\title{
Potentially pathogenic Acanthamoeba genotype T4 isolated from dental units and emergency combination showers
}

\author{
Esteban Castro-Artavia ${ }^{1,2}$, Lissette Retana-Moreira ${ }^{1,2}$, \\ Jacob Lorenzo-Morales ${ }^{3}$, Elizabeth Abrahams-Sandî́,2/+ \\ ${ }^{1}$ University of Costa Rica, Faculty of Microbiology, Department of Parasitology, San Pedro, San José, Costa Rica \\ ${ }^{2}$ University of Costa Rica, Centro de Investigación en Enfermedades Tropicales, San Pedro, San José, Costa Rica \\ ${ }^{3}$ University of La Laguna, Institute of Tropical Diseases and Public Health of the Canary Islands, Tenerife, Spain
}

BACKGROUND Acanthamoeba is the genus of free-living amoebae that is most frequently isolated in nature. To date, 20 Acanthamoeba genotypes have been described. Genotype T4 is responsible for approximately $90 \%$ of encephalitis and keratitis cases. Due to the ubiquitous presence of amoebae, isolation from environmental sources is not uncommon; to determine the clinical importance of an isolation, it is necessary to have evidence of the pathogenic potential of amoebae.

OBJECTIVE The aim of this study was to physiologically characterise 8 Acanthamoeba T4 isolates obtained from dental units and emergency combination showers and to determine their pathogenic potential by employing different laboratory techniques.

METHODS Eightaxenic cultures of Acanthamoeba genotype T4 were used in pathogenic potential assays. Osmotolerance, thermotolerance, determination and characterisation of extracellular proteases and evaluation of cytopathic effects in MDCK cells were performed.

FINDINGS All of the isolates were osmotolerant, thermotolerant and had serine proteases from 44-122 kDa. Two isolates had cytopathic effects on the MDCK cell monolayer.

MAIN CONCLUSION The presence of Acanthamoeba T4 with pathogenic potential in areas such as those tested in this study reaffirms the need for adequate cleaning and maintenance protocols to reduce the possibility of infection with free-living amoebae.

Key words: Acanthamoeba genotype T4 - free-living amoeba - dental unit - emergency combination showers

Acanthamoeba is a free-living amoebae (FLA) of worldwide distribution, commonly found in soil and water sources. Based on rRNA sequences, this genus is divided into 20 different genotypes (Corsaro et al. 2015), known as T1 to T20. Genotype T4 is the most frequently isolated genotype from clinical cases (Khan 2006, Maciver et al. 2013). It has been reported that more than $90 \%$ of amoebic keratitis cases have been linked with this genotype, with similar percentages for granulomatous amoebic encephalitis and cutaneous infections (Khan 2006). Other isolated genotypes linked to clinical manifestations include T2, T3, T5, T6, T11 and T15 (Di Cave et al. 2008, Walochnik et al. 2008, Ledee et al. 2009, Lorenzo-Morales et al. 2011, Omaña-Molina et al. 2016). In addition to their capacity to produce damage, some Acanthamoeba trophozoites harbour pathogenic bacteria, which are resistant to the lytic mechanisms of the amoeba. Reports include Legionella sp, Franciscella tularensis, nontuberculous mycobacteria, and others (Greub \& Raoult 2004). In fact, it has been postulated

doi: 10.1590/0074-02760170147

Financial support: Vicerrectoría de Investigación, University of Costa Rica (project 803-B4-050). JLM was supported by the grants RICET (Project no. RD12/0018/0012), Spanish Ministry of Health, Madrid, Spain (Project PI13/00490 from the Instituto de Salud Carlos III), Project ref. AGUA3 from Caja Canarias Fundación, and the Ramón y Cajal Subprogramme from the Spanish Ministry of Economy and Competitivity (RYC-2011-08863).

+Corresponding author: elizabeth.abrahams@ucr.ac.cr

Received 13 April 2017

Accepted 20 June 2017 that amoebae could be considered biological incubators or environmental reservoirs of these bacteria, acting as "Trojan horses" and increasing the transmission potential for humans (Janda 2010).

Due to the ubiquitous presence of amoebae, isolation from environmental sources is common. To date, several studies report Acanthamoeba in soil (especially genotype T4), swimming pools, water networks from hospitals, and air conditioning units (Thomas et al. 2006, Chan et al. 2011, Fabres et al. 2016, Reyes-Batlle et al. 2016), as well as equipment and devices for medical treatments or emergency situations. This equipment includes dialysis and dental units (Dendana et al. 2008, Trabelsi et al. 2010, Retana-Moreira et al. 2015), as well as combination shower units (Retana-Moreira et al. 2014).

Different laboratory techniques have been employed to demonstrate the presence of characteristics considered necessary for the amoebae to be potentially pathogenic (Khan 2006). Some of the techniques include thermotolerance and osmotolerance assays, as well as the secretion of extracellular proteases and the evaluation of cytopathic effects in cell culture. In this study, we employed these techniques in eight axenic isolates of Acanthamoeba T4 from dental and emergency combination showers to determine their pathogenic potential.

\section{MATERIALS AND METHODS}

Samples - A total of eight axenic isolates of Acanthamoeba genotype T4 were employed in this study. Samples were obtained from dental units (DU3, DU4, DU5, DU6, DU8, DU12) (Retana-Moreira et al. 2015) and emergency combination showers (CSU7 and CSUT7) (Retana- 
Moreira et al. 2014). Each axenic isolate was grown on $25 \mathrm{~cm}^{2}$ tissue culture flasks (Cellstar, Greiner Bio-One $\mathrm{GmbH}$, Frickenhausen, Germany) with $5 \mathrm{~mL}$ of PYG medium $(0.75 \%$ proteose peptone, $0.75 \%$ yeast extract and $1.5 \%$ glucose) containing $10 \mu \mathrm{g} / \mathrm{mL}$ gentamicin (Sigma Aldrich Co., St. Louis, USA). Isolates were maintained under aerobic conditions at $30^{\circ} \mathrm{C}$ without shaking.

Acanthamoeba Neff (ATCC30010) and the isolate CLC-16 genotype T3 (Martín-Navarro et al. 2010) were used as positive controls.

Osmotolerance and thermotolerance assays - The amoebae were cultivated on non-nutrient agar plates with $0.5 \mathrm{M}$ and $1 \mathrm{M}$ mannitol, supplemented with Escherichia coli. Approximately 1000 trophozoites were separately inoculated at the centre of the agar plate and incubated at $30^{\circ} \mathrm{C}$ for up to $72 \mathrm{~h}$ with daily observation. Proliferation of Acanthamoeba sp. was observed by measuring the increase in diameter of the clearance zone in the bacterial lawn.

For thermotolerance assays, the same quantity of amoebae was inoculated onto non-nutrient agar plates supplemented with $E$. coli. Cultures were incubated at $37^{\circ} \mathrm{C}$ and $40^{\circ} \mathrm{C}$. Proliferation of Acanthamoeba sp. was evaluated by inverted microscopic examination daily for up to $72 \mathrm{~h}$. Each assay was performed in triplicate.

Preparation of Acanthamoeba conditioned medium $(A C M)$ - ACM was prepared by incubating amoebae confluent flasks with $5 \mathrm{~mL}$ of RPMI-1640 medium (Sigma Aldrich Co., St. Louis, USA) at $30^{\circ} \mathrm{C}$ for $24 \mathrm{~h}$. After this time, cell-free conditioned medium was collected by centrifugation at $15000 \mathrm{rpm}$ for $10 \mathrm{~min}$, filtered through a 0.22 $\mu \mathrm{m}$ pore filter (Minisart, Sartorius stedim Biotech $\mathrm{GmbH}$, Goettingen, Germany) and stored at $-80^{\circ} \mathrm{C}$ until use.

Determination and characterisation of protease secretion by zymography - Zymographic assays were performed as previously described by Herron et al. (1986). Briefly, ACM was mixed with the sample buffer $(10 \%$ sodium dodecyl sulphate, $4 \%$ sucrose, $0.25 \mathrm{mM}$ Tris- $\mathrm{HCl}$ and $0.1 \%$ bromofenol, $\mathrm{pH}$ 6.8) and underwent electrophoresis on SDS-polyacrylamide gels (SDS-PAGE) containing gelatin $(1 \mathrm{mg} / \mathrm{mL})$. After electrophoresis, gels were washed twice in $2.5 \%$ Triton X-100 (w/v) for $30 \mathrm{~min}$ to remove the SDS. Finally, gels were incubated overnight, at $37^{\circ} \mathrm{C}$, in a developing buffer $(50 \mathrm{mM}$ Tris-HCl, $\mathrm{pH} 8.0$, containing $10 \mathrm{mM}$ $\mathrm{CaCl}_{2}$ ), and rinsed and stained with Coomassie brilliant blue R-250 (Bio-Rad Laboratories, California, USA). Areas of gelatin digestion, which indicate protease activity, were seen as non-staining regions in the gel.

To characterise the type of protease produced, samples were pre-treated for 30 minutes with phenylmethylsulfonyl fluoride (PMSF, $1 \mathrm{mM}$ final concentration) or 1, 10-phenanthroline (10 mM final concentration) (Sigma Aldrich Co., St. Louis, USA) before electrophoresis. As 1,10-phenanthroline is a reversible inhibitor, it was also included in the developing buffer.

Evaluation of the in vitro effect of Acanthamoeba on cell culture - Cell culture: Madin-Darby canine kidney (MDCK) epithelial cells (NBL2 ATCC CCL-34TM) were grown in $75 \mathrm{~cm}^{2}$ cell culture flasks (Corning, Corning
Incorporated, NY, USA) containing RPMI 1640 medium (Sigma-Aldrich Co., ST. Louis, USA) with penicillin (100 $\mathrm{U} / \mathrm{mL})$, streptomycin $(100 \mathrm{pg} / \mathrm{mL})$ and $10 \%$ foetal calf serum (Gibco, GranIsland, NY, USA). Flasks were maintained at $37^{\circ} \mathrm{C}$ in a humidified $5 \% \mathrm{CO}_{2}$ incubator.

Crystal violet stain: MDCK cells were grown until confluence on 24-well plates. Then, Acanthamoeba isolates $\left(5 \times 10^{5}\right.$ amoebae per well) were incubated with cell monolayers in serum-free media for $24 \mathrm{~h}$ at $37^{\circ} \mathrm{C}$. The cytopathic effect was assessed macroscopically after crystal violet staining of the wells. The strains Acanthamoeba castellanii Neff American Type Culture Collection 30010) and Acanthamoeba T3 strain CLC-16 (Martín-Navarro et al. 2010) were employed as positive controls.

Cytotoxicity assay: MDCK cells were incubated with each Acanthamoeba isolate as described above. At the end of the incubation period, supernatants were collected. Cytotoxicity was determined by measuring lactate dehydrogenase $(\mathrm{LDH})$ release at $590 \mathrm{~nm}$, following the indications of the commercial kit manufacturers (LDH BR, Linear Chemicals, Barcelona, Spain).

The percentage of LDH released (\% cytotoxicity) was calculated as follows: [LDH activity in experimental sample (measured by optical density at $590 \mathrm{~nm}$ ) - LDH activity in control samples]/(total LDH activity release LDH activity in control samples) $\times 100$. Control sample values were obtained from host cells incubated in RPMI 1640. Total LDH release was determined from host cells treated with $1 \%$ Triton $\mathrm{X}-100$ for $30 \mathrm{~min}$ at $37^{\circ} \mathrm{C}$.

Strains A. castellanii Neff (ATCC) 30010 and Acanthamoeba T3 strain CLC-16 (Martín-Navarro et al. 2010) were employed as positive controls.

\section{RESULTS}

The eight axenic isolates employed in this study were able to grow in plates with $0.5 \mathrm{M}$ and $1.0 \mathrm{M}$ mannitol. The thermotolerance assay showed the same result, as $100 \%$ of the isolates grew when incubated at temperatures of $37^{\circ} \mathrm{C}$ and $40^{\circ} \mathrm{C}$.

Regarding the secretion of extracellular proteases, ACM from all Acanthamoeba isolates showed similar banding patterns on gelatin gels (Fig. 1). Four bands, with molecular weights of approximately 44, 46, 58 and $124 \mathrm{kDa}$, were observed. All proteases were inhibited when incubating with PMSF $1 \mathrm{mM}$. In contrast, none of the bands were inhibited by 1, 10-phenanthroline 10 $\mathrm{mM}$. The results suggest the secretion of serine proteases in all isolates.

An apparent damage of the cell monolayer was observed only after incubation of the cells with isolates CSUT7 and DU12. The remaining isolates did not have significant monolayer alterations. Fig. 2 shows the crystal violet staining of cells incubated with the Acanthamoeba isolates mentioned above, as well as the results of the control strains.

In the LDH release assay, six isolates showed a percentage $\leq 10 \%$. For isolates CSUT7 and DU12, the obtained percentages were $24.4 \%$ and $14.4 \%$, respectively. Control strains Acanthamoeba Neff and CLC-16 showed percentages of cytotoxicity of $60.9 \%$ and $38.8 \%$, respectively. 


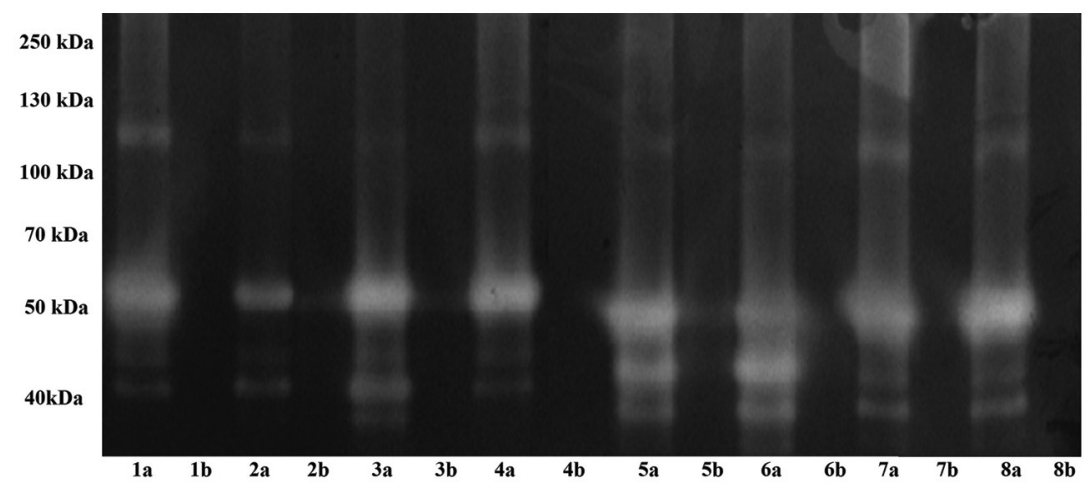

Fig. 1: protease zymograms of the eight environmental Acanthamoeba isolates. Lanes (a) Acanthamoeba conditioned medium (ACM); lanes (b) ACM incubated with serine protease inhibitor [phenylmethylsulfonyl fluoride (PMSF)]. 1: DU12; 2: DU5; 3: DU3; 4: DU8; 5: CSUT7; 6: CSU7; 7: DU6; 8: DU4.

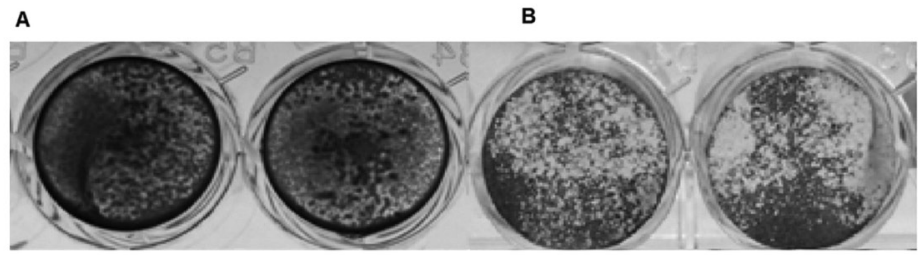

c

D

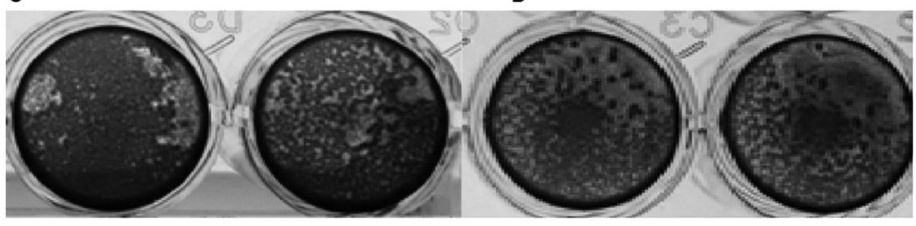

E

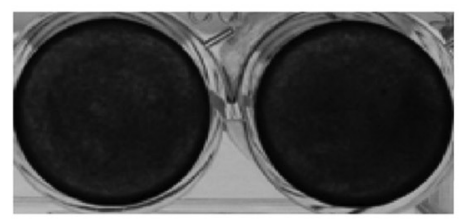

Fig. 2: crystal violet staining showed the cytopathic effect of three Acanthamoeba environmental isolates on Madin-Darby canine kidney (MDCK) cells. Amoebae were incubated in 24-multiwell plates containing MDCK for $24 \mathrm{~h}$ at $37^{\circ} \mathrm{C}$. (A) CSUT7; (B) control strain Acanthamoeba Neff; (C) DU12; (D) control strain Acanthamoeba CLC-16; (E) cell control. Photos are representative of triplicate experiments.

\section{DISCUSSION}

Acanthamoeba is the genus of FLA most frequently found in nature; nevertheless, not every species or genotype is able to produce damage to a host (Schuster \& Visvesvara 2004). In previous studies performed in our laboratory, we isolated and axenically grew eight Acanthamoeba genotype T4 from dental units and combined emergency units (Retana-Moreira et al. 2014, 2015). Considering the location from which they were isolated and, since this genotype is the most frequently associated with clinical cases (Khan 2006), we considered it relevant to physiologically and biologically characterise the isolates through a series of laboratory assays to determine characteristics associated with pathogenic potential.

The results showed that all Acanthamoeba genotype T4 employed in this study were osmotolerant and thermo- tolerant, which are indirect factors related to pathogenicity (Khan 2001). Growth at high mannitol concentrations has been associated with the ability to resist high osmotic pressures, a situation that the amoebae could face when they act as parasites of the corneal epithelium (Siddiqui \& Khan 2012). Thermotolerance is related to the ability of the amoeba to resist normal body temperature or even fever episodes in the host. Moreover, the growth of amoebae at temperatures above $40^{\circ} \mathrm{C}$ is directly correlated to their capacity to produce cellular damage in vitro (Griffin 1972, Walochnik et al. 2000). Even if these two characteristics give the amoeba adaptative advantages when parasitising a host, the mere presence or absence of these characteristics is not enough to define the amoeba as pathogenic. For instance, different species of Acanthamoeba may be thermotolerant but non-pathogenic (Schuster 
\& Visvesvara 2004). This characteristic is also less indicative of pathogenicity for amoebae that infect the human cornea because the corneal temperature is approximately $32-35^{\circ} \mathrm{C}$; therefore, colonisation by the amoeba could be possible even when the organism is not able to grow at temperatures above $37^{\circ} \mathrm{C}$ (Tolero et al. 2011).

In this study, we demonstrated the production of extracellular serine proteases by all of the environmental isolates. Migration patterns showed bands with molecular weights from $44 \mathrm{kDa}$ to $124 \mathrm{kDa}$. These results are similar to the ones obtained for other Acanthamoeba, including isolates from clinical cases (Cao et al. 1998, Alfieri et al. 2000). Early reports considered serine proteases as pathogenicity markers (Khan et al. 2000), with proven action of proteases in plasminogen activation, as well as collagen and fibronectin degradation (Khan 2006). Recently, the role of proteases in tissue invasion has been confirmed, emphasising their participation in extracellular matrix digestion but not in the direct process of cellular lysis (Omaña-Molina et al. 2013). It is important to highlight that the activity of these enzymes has been demonstrated particularly in Acanthamoeba isolated from clinical cases of encephalitis or keratitis. For environmental isolates (such as the ones employed in this study), a role of proteases in nutrition and encystment/excystment has been proposed (Dudley et al. 2008), considering their presence as necessary for eventual facultative parasitism by the amoeba.

To evaluate the possible in vitro cytotoxicity and cytopathic effect of our isolates, we used MDCK cells, a model of cytolytic activity frequently employed in this type of study. By crystal violet staining, it was possible to observe damage to the monolayer $24 \mathrm{~h}$ after adding DU12 and CSUT7 isolates or the control strains, A. castellani Neff and Acanthamaoeba CLC-16. The main observed effect was the disaggregation of the monolayer by amoebae trophozoites that were attached to the plate in the spaces previously occupied by cells or between the attached cells. When using the LDH release assay, these same isolates and control strains showed a level of cytotoxicity that correlated with the degree of cellular damage observed in the crystal violet assay. In a previous study employing different cell lines, Martín-Navarro et al. (2010) reported percentages of cytotoxicity for Acanthamoeba Neff of $60 \%$ to $70 \%$ and for CLC- $16,55 \%$ to $75 \%$. These percentages, especially for CLC-16, were significantly higher than those obtained in our study, which could be directly related to the use of the MDCK line. Despite the above mentioned study, and considering that Acanthamoeba Neff has a degree of cytotoxicity which could be categorised as moderate to high, our results suggest that the environmental isolates DU12 and CSUT7 showed an in vitro cytopathic effect that, although mild, reveals the capability of the amoebae to damage the cell monolayer.

Our study demonstrated virulence factors that suggest a pathogenic potential for all of the Acanthamoeba genotype T4 environmental isolates: osmotolerance, thermotolerance and secretion of extracellular proteases. Additionally, two of them (one from a dental unit and one from an emergency combined shower) showed in vitro cytopathic effects. The isolation of these types of amoebae in equipment and biosafety devices such as those tested in this study reaffirms the need for adequate cleaning and maintenance protocols to reduce the possibility of infection with FLA.

\section{AUTHORS' CONTRIBUTION}

LRM and EAS - Conceived the study; ECA, LRM, JLM and EAS - participated in the design of the study and physiological characterisation of amoebas; JLM, EAS and LRM revised the manuscript.

\section{REFERENCES}

Alfieri SC, Correia CEB, Motegi SA, Pral EMF. Proteinase activities in total extracts and in medium conditioned by Acanthamoeba polyphaga trophozoites. J Parasitol. 2000; 86(2): 220-7.

Cao Z, Jefferson DM, Panjwani N. Role of carbohydrate-mediated adherence in cytopathogenic mechanisms of Acanthamoeba. J Biol Chem. 1998; 273(25): 15838-45.

Chan LL, Mak JW, Low YT, Koh TT, Ithoi I, Mohamed SM. Isolation and characterization of Acanthamoeba spp. from air-conditioners in Kuala Lumpur, Malaysia. Acta Trop. 2011; 117(1): 23-30.

Corsaro D, Walochnik J, Köhsler M, Rott MB. Acanthamoeba misidentification and multiple labels: redefining genotypes T16, T19, and T20 and proposal for Acanthamoeba micheli sp. (genotype T19). Parasitol Res. 2015; 114(7): 2481-90.

Dendana F, Sellami H, Jarraya F, Sellami A, Makni F, Cheikhrouhou F, et al. Free-living amoebae (FLA): detection, morphological and molecular identification of Acanthamoeba genus in the hydraulic system of an haemodialysis unit in Tunisia. Parasite. 2008; 15(2): 137-42.

Di Cave D, Monno R, Bottalico P, Guerriero S, D’Amelio S, D’Orazi C, et al. Acanthamoeba T4 and T15 genotypes associated with keratitis infections in Italy. Eur J Clin Microbiol Infect Dis. 2008; 28(6): 607-12.

Dudley R, Alsam S, Khan NA. The role of proteases in the differentiation of Acanthamoeba castellanii. FEMS Microbiol Lett. 2008; 286(1): 9-15.

Fabres LF, dos Santos SPR, Benitez LB, Rott MB. Isolation and identification of Acanthamoeba spp. from thermal swimming pools and spas in Southern Brazil. Acta Parasitol. 2016; 61(2): 221-7.

Greub G, Raoult D. Microorganisms resistant to free-living amoebae. Clin Microbiol Rev. 2004; 17(2): 413-33.

Griffin JL. Temperature tolerance of pathogenic and nonpathogenic free-living amoebas. Science. 1972; 178(4063): 869-87.

Herron GS, Banda MJ, Clark EJ, Gavrilovic J, Werb Z. Secretion of metalloproteinases by stimulated capillary endothelial cells. J Biol Chem. 1986; 261(6): 2814-8.

Janda WM. Amoeba-resistant bacteria: their role in human infections. Clin Microbiol News. 2010; 32(23): 177-84.

Khan NA, Jarroll EL, Panjwani N, Cao Z, Paget TA. Proteases as markers for differentiation of pathogenic and nonpathogenic species of Acanthamoeba. J Clin Microbiol. 2000; 38(8): 2858-61.

Khan NA. Acanthamoeba: biology and increasing importance in human health. FEMS Microbiol Rev. 2006; 30(4): 564-95.

Khan NA. Pathogenicity, morphology, and differentiation of Acanthamoeba. Curr Microbiol. 2001; 43(6): 391-5.

Ledee DR, Iovieno A, Miller D, Mandal N, Diaz M, Fell J, et al. Molecular identification of T4 and T5 genotypes in isolates from Acanthamoeba keratitis patients. Clin Microbiol. 2009; 47(5): 1458-62. 
Lorenzo-Morales J, Morcillo-Laiz R, Martín-Navarro CM, López-Vélez R, López-Arencibia A, Arnalich-Montiel F, et al. Acanthamoeba keratitis due to genotype T11 in a rigid gas permeable contact lens wearer in Spain. Cont Lens Anterior Eye. 2011; 34(2): 83-6.

Maciver SK, Asif M, Simmen MW, Lorenzo-Morales J. A systematic analysis of Acanthamoeba genotype frequency correlated with source and pathogenicity: T4 is confirmed as a pathogen-rich genotype. Eur J Protistol. 2013; 49(2): 217-21.

Martín-Navarro CM, Lorenzo-Morales J, Machín RP, López-Arencibia A, Valladares B, Piñero JE. Acanthamoeba spp.: in vitro effects of clinical isolates on murine macrophages, osteosarcoma and HeLa cells. Exp Parasitol. 2010; 126(1): 85-8.

Omaña-Molina M, González-Robles A, Salazar-Villatoro LI, LorenzoMorales J, Cristóbal-Ramos AR, Hernández-Ramírez VI, et al. Reevaluating the role of Acanthamoeba proteases in tissue invasion: observation of cytopathogenic mechanisms on MDCK cell monolayers and hamster corneal cells. Biomed Res Int. 2013; 461329.

Omaña-Molina M, Vanzzini-Zago V, Hernández-Martínez D, González-Robles A, Salazar-Villatoro L, Ramírez-Flores E, et al. Acanthamoeba genotypes T3 and T4 as causative agents of amoebic keratitis in Mexico. Parasitol Res. 2016; 115(2): 873-8.

Retana-Moreira L, Abrahams-Sandí E, Cabello-Vílchez AM, ReyesBatlle M, Valladares B, Martínez CE, et al. Isolation and molecular characterization of Acanthamoeba and Balamuthia mandrillaris from combination shower units in Costa Rica. Parasitol Res. 2014; 113(11): 4117-22.

Retana-Moreira L, Abrahams-Sandí E, Castro-Artavia E, FernándezSánchez A, Castro-Castillo A, Reyes-Batlle M, et al. Isolation and molecular characterization of Acanthamoeba strains from dental units in Costa Rica. J Eukaryot Microbiol. 2015; 62(6): 733-6.
Reyes-Batlle M, Zamora-Herrera J, Vargas-Mesa A, Valerón-Tejera MA, Wagner C, Martín-Navarro CM, et al. Acanthamoeba genotypes T2, T4, and T11 in soil sources from El Hierro Island, Canary Islands, Spain. Parasitol Res. 2016; 115(8): 2953-6.

Schuster FL, Visvesvara GS. Free-living amoebae as opportunistic and non-opportunistic pathogens of humans and animals. Int $\mathrm{J}$ Parasitol. 2004; 34(9): 1001-27.

Siddiqui R, Khan NA. Biology and pathogenesis of Acanthamoeba. Parasit Vectors. 2012; 5: 1-6.

Thomas V, Herrera-Rimann K, Blanc DS, Greub G. Biodiversity of amoebae and amoeba-resisting bacteria in a hospital water network. Appl Environ Microbiol. 2006; 72(4): 2428-38.

Tolero W, Caumo K, Brittes RM. Prevalence of Acanthamoeba from tap water in Rio Grande do Sul, Brazil. Curr Microbiol. 2011; 63(5): 464-9.

Trabelsi H, Sellami A, Dendana F, Sellami H, Cheikh-Rouhou F, Makni F, et al. Free-living amoebae (FLA): morphological and molecular identification of Acanthamoeba in dental unit water. Parasite. 2010; 17(1): 67-70.

Walochnik J, Aichelburg A, Assadian O, Steuer A, Visvesvara G, Vetter N, et al. Granulomatous amoebic encephalitis caused by Acanthamoeba amoebae of genotype $\mathrm{T} 2$ in a human immunodeficiency virus-negative patient. J Clin Microbiol. 2008; 46(1): 338-40.

Walochnik J, Obwaller A, Aspöck H. Correlations between morphological, molecular biological and physiological characteristics in clinical and nonclinical isolates of Acanthamoeba spp. Am Soc Microbiol. 2000; 66(10): 4408-13. 